\title{
Simulation of Fingering Behavior in Smoldering Combustion Using a Cellular Automaton
}

\author{
Nieves Fernandez-Anez ${ }^{\mathrm{a}, \mathrm{b}, \mathrm{c}, \mathrm{d}}$, Kim Christensen ${ }^{\mathrm{a}, \mathrm{b}}$, Vidar Frette $^{\mathrm{d}}$ and Guillermo Rein ${ }^{\mathrm{c}}$ \\ ${ }^{a}$ Centre for Complexity Science, Imperial College London, SW7 2AZ, London, United Kingdom. \\ ${ }^{\mathrm{b}}$ Blackett Laboratory, Imperial College London, SW7 2AZ, London, United Kingdom. \\ ${ }^{c}$ Department of Mechanical Engineering, Imperial College London, SW7 2AZ, United Kingdom. \\ ${ }^{\mathrm{d}}$ Department of Fire Safety and HSE Engineering, Western Norway University of Applied Sciences.
}

Receipt date: $7^{\text {th }}$ July 2018

\begin{abstract}
Smoldering is the slow, low-temperature, flameless burning of porous fuels and the most persistent type of combustion phenomena. It is a complex physical process that is not yet completely understood, but it is known that it is driven by heat transfer, mass transfer and fuel chemistry. A specific case of high interest and complexity is fingering behavior. Fingering is an instability that occurs when a thin fuel layer burns against an oxygen current. These instabilities appear when conduction rather than convection is the dominant mode of heat transfer to the fuel ahead and the availability of oxygen is limited during the combustion of a thin fuel, such as paper. The pattern of the fingers can be characterized through the distance between them and their width, and can be classified into three different regimes: isolated fingers, tip-splitting fingers or no finger form, a smooth continuous front. In this paper, a multi-layer cellular automaton based on three governing principles (heat, oxygen and fuel) is shown to reproduce all the regimes and the details of finger structures observed in previous experiments. It is shown how when oxygen is not limited, a smooth smoldering front is formed. If the oxygen speed decreases beyond a critical value, fingers appear first as tip-splitting fingers and later as isolated fingers, increasing the distance between them and decreasing their thickness. The oxygen consumed during oxidation influences these critical values with a positive correlation. This cellular automaton provides a novel approach to simulate smoldering combustion in large systems over long time. That the model is able to reproduce the complex pattern formation seen in a fingering experiment validates the model. In the future, we could apply the model in various other geometries to make predictions on the outcome smoldering combustion processes.
\end{abstract}

\section{INTRODUCTION}

In many situations, as observed in the built environment, a distinct physical front moves through a medium. At the front, a relevant variable (e.g. a concentration) changes abruptly. As it spreads, the front may maintain a simple shape - or develop increasingly complex (even fractal) geometries.

A classical and illustrative example of this is viscous fingering, where one fluid displaces another. If the two fluids are immiscible, a sharp front is formed. Depending on viscosities, the front may be simple or complex. This is illustrated in the case of a thin fluid layer between two horizontal plates, known as Hele-Shaw apparatus $[1,4]$ in Fig. 1. With the invading fluid injected at the central point and the cell open to the atmosphere at the rim, the advancing front may be simple (smooth shape) or complex (fingering behavior). When the more viscous fluid is injected and displaces the less viscous fluid, the front is simple (left of Fig. 1). In the opposite case, the front is complex and forms a finger structure 
(right of Fig. 1). The injection of less viscous fluid creates a pressure field that decreases from the center through the two fluids. At the tip of any small finger or front disturbance, the radial distance through the more viscous fluid to the rim of the cell is shorter than elsewhere. Since the pressure fall in the less viscous fluid is small, the pressure gradient at finger tips is large, and, as a consequence, the fingers spread more rapidly than other parts of the front. Similar mechanisms for this spread of small disturbances and fingers are at work in other physical processes.
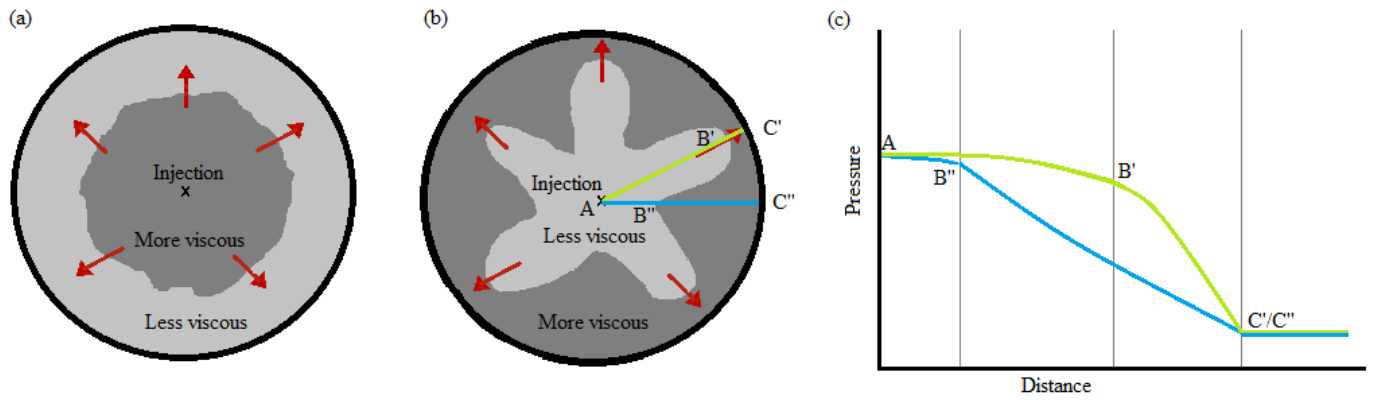

FIG. 1. Schematic of the evolution of the front formed when a fluid is injected at the central point of a circular Hele-Shaw apparatus filled by another fluid. (a) The injected fluid is more viscous than the one in the apparatus.

The front is stable. (b) The injected fluid is less viscous than the one in the apparatus. The front is unstable;

fingers are formed. (c) Schematic pressure distributions along two radii, one passing through a finger [green (upper)] and the other passing in between two fingers [blue (lower)].

The situation in Fig. 1 is reversible, it is possible to reverse the flow direction and take both fluids back to the initial condition. In other cases, irreversible changes occur through chemical reaction, mixing or similar. An example is the front (edge) of a colony of bacteria spreading in a substrate that contains nutrients [5].

Here we consider the irreversible process of combustion, where the front spreads through unburnt material, leaving behind burnt material. The particular combustion process that can originate fingers is smoldering, a slow, low-temperature flameless form of combustion [6], which is sustained by the heat generated when oxygen reacts directly at the surface of a condensed-phase fuel. Smoldering is governed by heat transfer and oxygen supply, with the chemical kinetics playing a secondary role [7].

Fingering instabilities in smoldering combustion fronts appear under specific conditions. Under atmospheric conditions, a thin fuel layer, opposed laminar oxygen flow and conduction as the dominant heat transfer mode are the essential conditions for observing fingering behavior.

Zik et al. observed this behavior in the late 90s [8], concluding that there is a dependence between the Peclet number ( $\mathrm{Pe}$ ) (which is proportional to the speed of the imposed oxygen flow) and the distance between fingers. The authors established that above a critical value of Pe, the front is smooth. As Pe is slightly decreased, small bumps along the interface begin to compete over the oxygen, and the front develops a more complicated structure. This marks the onset of fingers. When the oxygen supply is further decreased, lateral diffusion becomes more important and the bumps become periodic in the space along the front. As the oxygen supply is further decreased, the small peaks of the periodic structure deplete the oxygen in their vicinity, and fingers are formed.

Zik et al. concluded without proof that the width of the fingers depends on the heat release on the front. Recently, Kuwana et al. [9] experimentally proved this hypothesis relating the width of the fingers with the Lewis number (ratio of thermal diffusivity to mass diffusivity) and showing that the higher the oxygen speed is, the wider the fingers are.

Iijoma et al. [10] developed a model that simulates this behavior through a one-step reaction, and obtained the patterns that can appear under different conditions. Later, the authors also demonstrated 
the influence of non-uniformity in the fuel sample, where the direction of the fingers may not necessarily be in the direction of the oxidizer flow [11].

In this paper, we are presenting a cellular automaton model that reproduces the fingering behavior in smoldering combustion. Cellular automaton is an ideal methodology for modelling emergent complex processes through simple rules [12]. It has been considered a suitable alternative to differential equations for many physical systems and processes. This is a stochastic and non-dimensional model based on a three-step reaction scheme. The cellular automata reproduces the patterns observed in the experiments and the numerical results show the importance of the oxygen speed and the heat transfer on the appearance and development of the fingering behavior.

Cellular automaton can be used to study the spread of smoldering fires and provides enough accuracy to observe the influence of ignition location and direction of airflow in this spread. The model was conceived as the simplest mathematical model able to reproduce smoldering fires. Fingering behavior on smoldering fronts has been used as a reference case to test the accuracy of the cellular automaton with a well-known and controlled experimental setup.

This paper is divided in 6 sections. After the introduction, we present the cellular automata used to model the fingering process. In this second section, the multi-layer model is presented including the rules that each of the layer follows and the relations between the different layers. This section ends with an explanation of the parameters used in the model and their numerical values. The third section explains the influence of the oxygen speed on the appearance of fingers in a fire front, showing how by decreasing the forced oxygen flow the pattern vary from a smooth continues front to simple fingers. Section IV shows how the amount of oxygen consumed during oxidation modifies the patterns that appear during the combustion. After this, a numerical results section show the relations existing between both the oxygen speed and the oxygen consumption and the two main characteristics of the fingers: distance between fingers and their width. This paper finishes with a section presenting the concluding remarks.

\section{METHODOLOGY}

Two-dimensional cellular automata are discrete dynamic systems, composed of a finite number of identical cells, arranged uniformly in a two-dimensional space [13]. They have an associated state that could change at every step of time according to pre-fixed rules, mimicking the relevant processes.

The cellular automaton used in this paper models a smoldering process spreading across a rectangular sheet of paper in a Hele-Shaw apparatus, as shown in Fig. 2. It describes the dynamics of smoldering fronts using a three-layer approach that takes into account the two mechanisms controlling the spread of smoldering - oxygen supply and heat transfer - and the changes that occur in the proper fuel [14]. All the parameters that characterize the model are non-dimensional. For example, a time step is defined as updating all cells once. This model is based in a previous work from the authors [14] where the first version of the smoldering cellular automaton was presented. The new version of the model, presented here, has a more realistic heat layer that includes radiation and convection processes, and also included the oxygen shadow dimensions that are essential for the process. 


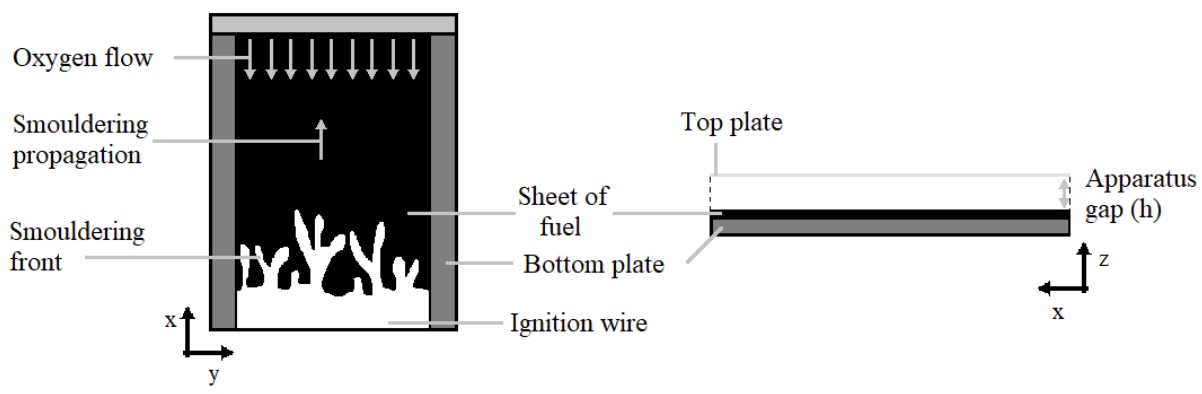

FIG. 2. Schematic of the experiments in a Hele-Shaw apparatus that are modeled by the cellular automaton. Top (left) and side (right) views. Notice that oxygen flow is opposite to the propagation of the smoldering front.

The experimental setup that is replicated in this research [8] consists of a Hele-Shaw apparatus with an adjustable space $h$ between the sample plane and the glass top. Paper was the chosen fuel by Zik et al. due to its high uniformity, the amount of information available on its combustion behavior, and the fact that its thickness is small enough compared to the other dimensions to be considered as twodimensional. An oxygen flow was imposed from one edge, at controlled speed. Combustion was initiated at the other edge, using an ignition wire. The oxygen flow and the combustion front move in opposite directions, thus, the experiment is a configuration for opposed smoldering. Fingering instabilities during combustion under atmospheric conditions are only observed in opposed mode, and not if oxygen and combustion front move in the same direction (forward smoldering).

Unlike other cellular automata models that use a single layer, the one presented in this manuscript uses multiple layers whose rules are interconnected and detailed in section D. In this section, we first describe each of the layers, and second, we describe the connections between them. A list of parameters (section II-E) and an explanation of the image processing (section II-F) completes this section on methodology.

\section{A. Fuel layer}

The fuel layer is the only one previously used in the different cellular automata fire models [15], [16]. It represents the chemical conversions taking place between chemical states. The mechanism controlling this layer is the three-step scheme that drives smoldering combustion [7]:

$$
\text { Fuel (wet) } \stackrel{\text { Drying }}{\longrightarrow} \text { Fuel (dry) } \stackrel{\text { Pyrolysis }}{\longrightarrow} \text { Char } \stackrel{\text { Oxidation }}{\longrightarrow} \text { Ash. }
$$

The parameters involved in this layer are the probability rates of the three processes: drying $\left(\mathrm{p}_{\mathrm{d}}\right)$, pyrolysis $\left(\mathrm{p}_{\mathrm{p}}\right)$ and oxidation $\left(\mathrm{p}_{\mathrm{o}}\right)$. Qualitatively, a high probability means that the corresponding process occurs easily and fast, while a low probability rate implies that the process is difficult and slow.

\section{B. Heat layer}

The heat layer represents heat exchanges that occur during smoldering, as described by the heat transfer equation for the fuel, represented by Fig. 3. The equations (rules) presented and the model described are a two-dimensional simplification that mimic the processes of a three-dimensional problem.

$$
\frac{\partial u(x, y, t)}{\partial t}=\gamma \nabla^{2} u(x, y, t)
$$

where $u(x, y, t)$ is the thermal energy, $\gamma$ is the overall heat transfer coefficient and $\nabla^{2}$ is the Laplace operator, which in Cartesian coordinates is given by $\nabla^{2}=\frac{\partial^{2}}{\partial x^{2}}+\frac{\partial^{2}}{\partial y^{2}}$ in two dimensions. 
This differential equation in terms of time, $t$, and space, $x$ and $y$, for a two-dimensional situation, is equivalent to:

$$
\begin{aligned}
& \lim _{\Delta t \rightarrow 0} \frac{u(x, y, t+\Delta t)-u(x, y, t)}{\Delta t}=\gamma \lim _{\Delta x \rightarrow 0} \frac{u(x+\Delta x, y, t)+u(x-\Delta x, y, t)-2 u(x, y, t)}{(\Delta x)^{2}} \\
&+\gamma \lim _{\Delta y \rightarrow 0} \frac{u(x, y+\Delta y, t)+u(x, y-\Delta y, t)-2 u(x, y, t)}{(\Delta y)^{2}} .
\end{aligned}
$$

Discretizing Equation 2 using finite differences

$$
\begin{gathered}
u(x, y, t+\Delta t)-u(x, y, t)=\gamma \frac{u(x+\Delta x, y, t)+u(x-\Delta x, y, t)-2 u(x, y, t)}{(\Delta x)^{2}} \Delta t \\
+\gamma \frac{u(x, y+\Delta y, t)+u(x, y-\Delta y, t)-2 u(x, y, t)}{(\Delta y)^{2}} \Delta t .
\end{gathered}
$$

Assuming that $\Delta x=\Delta y=\Delta t=1$

$$
\begin{aligned}
u(x, y, t+\Delta t)=u(x, y, t)+ & \gamma[u(x+\Delta x, y, t)+u(x-\Delta x, y, t)-2 u(x, y, t)] \\
& +\gamma[u(x, y+\Delta y, t)+u(x, y-\Delta y, t)-2 u(x, y, t)] .
\end{aligned}
$$

Additionally, the overall heat transfer coefficient $(\gamma)$ is the sum of the effects due to conduction and convection as: $\gamma=\gamma_{c d}+\gamma_{c v}$, where the conductive term $\left(\gamma_{c d}\right)$ is a constant number that represents the heat exchange inside the porous fuel including the radiative conductivity in the optically thick limit [17], and the convection term $\left(\gamma_{c v}\right)$ is directly related to the speed of the oxygen flow.

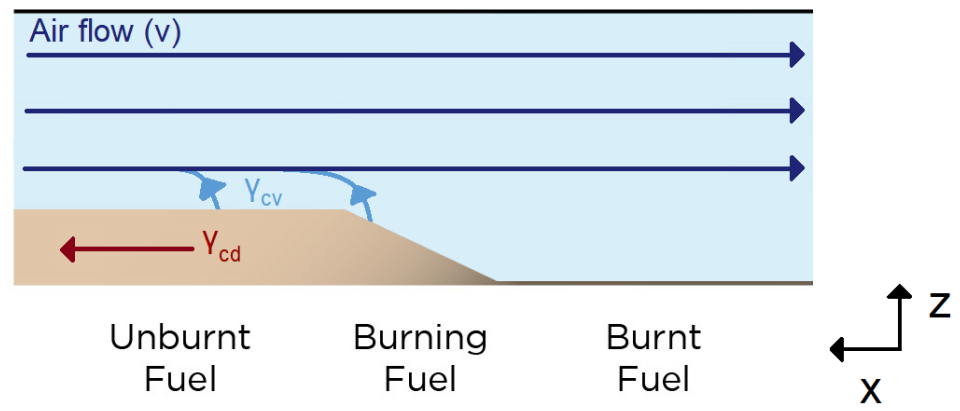

FIG. 3. Graphical explanation of the terms of the overall heat transfer parameter. The conductive term, $\gamma c d$, is directly related with the fuel, and has a constant value. The convection term, $\gamma c v$, depends on the speed of the oxygen flow and is assumed to be proportional to the square root of the oxygen speed.

Since the convection term is related to the speed of the oxygen, the values of the heat transfer parameters depend on the direction of the flow, being larger along the oxygen flow, and smaller in the opposite and perpendicular directions, as shown in Fig. 4.

The value of the heat parameter in the direction of the oxygen flow is: $\gamma_{f}=0.5+0.5 v^{1 / 2}[18]$, representing a laminar external flow over a flat plate, where $v$ is the oxygen speed. The heat transfer parameter in the opposite direction varies according to the expression $\gamma_{o}=0.2 \gamma_{f}$, and the parameter in both of the perpendicular directions is given by the expression: $\gamma_{l}=\frac{1}{2}\left(\gamma_{f}+\gamma_{o}\right)$

Once these parameters are defined, the discretized heat transfer equation should be changed to fit the two-dimensional grid of our domain (Fig. 4). If the smoldering front propagates in the positive $\mathrm{x}$ direction (see Fig. 3), the equation is as follows,

$$
u(x, y, t+\Delta t)=u(x, y, t)+
$$




$$
\begin{aligned}
+\gamma_{o}[u(x+\Delta x, y, t) & -u(x, y, t)]+\gamma_{f}[u(x-\Delta x, y, t)-u(x, y, t)]+ \\
+ & \gamma_{l}[u(x, y+\Delta y, t)+u(x, y-\Delta y, t)-2 u(x, y, t)] .
\end{aligned}
$$

When oxidation takes place, there is a punctual release of heat represented by the parameter $\Omega_{o}$. On the other hand, when drying or pyrolysis take place, a punctual consumption of heat occurs $\left(\Omega_{d}\right.$ or $\left.\Omega_{p}\right)$.

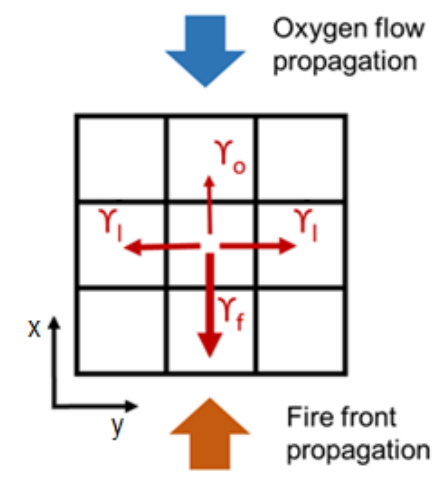

FIG. 4. Effective diffusivities (heat transfer parameters) in relation to the direction of the oxygen flow, for opposed propagation. The effective diffusivity on the opposite direction of the reaction front is larger than the one on the same direction.

\section{Oxygen layer}

The oxygen current above the fuel transports the oxygen needed for oxidation, and therefore interacts with the fuel layer. The parameter controlling the evolution of the oxygen front is the oxygen speed, defined by:

$$
v=\frac{\text { number of cells in the front that changes state from } 0 \text { to } 1 \text { per time step }}{\text { width of the system }}
$$

The oxygen flow is laminar because of the Hele-Shaw apparatus, which we represent using addition of oxygen-rich sites at randomly chosen positions of the oxygen front followed by lateral diffusion: addition at a randomly chosen site, a particle propagates one cell vertically. Lateral diffusion means that we allow the deposited particle to diffuse along the surface up to a finite distance. By the combination of both, the oxygen advances perpendicular to the ignition line. The flow of oxygen in a real system would never be deterministically uniform across the width of the system, so the random choice of the oxygen-rich sites is the simplest way of implementing it in the model. On a large scale (over long time) the flow will be uniform but with small fluctuations. The average speed of the oxygen flow is represented by the number of cells that the oxygen front advances through per time step and system width, as given in Equation (7) and explained in Fig. 5. 


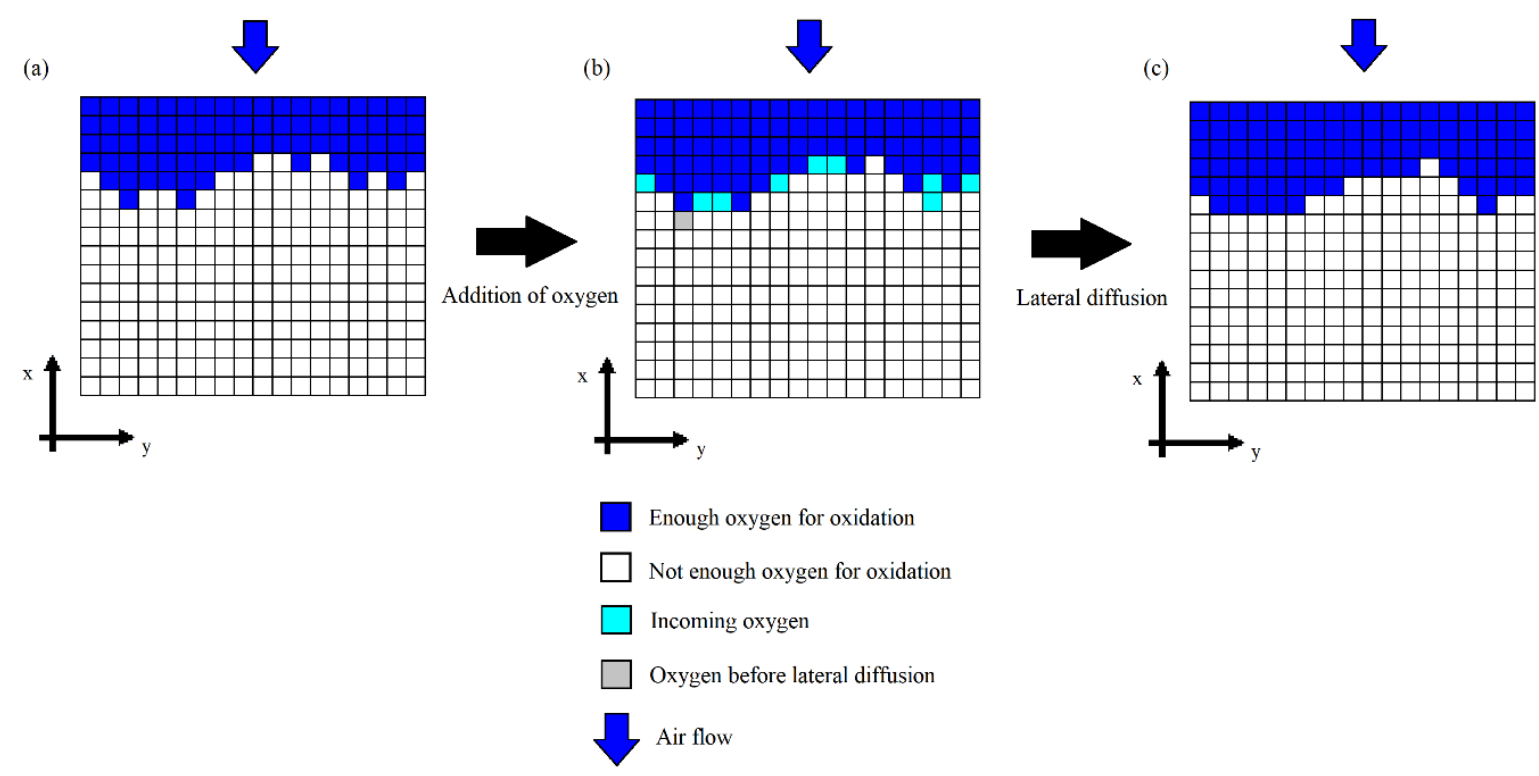

FIG. 5. Evolution of the oxygen flow, with ten cells per time step, corresponding to an oxygen speed of 10/18 0.56 layers per time step. The first step consists of the addition of ten new oxygen-rich cells in random locations at the oxygen front. The second step consists of lateral diffusion of oxygen-rich cells along the oxygen front. In (c), all new oxygen-rich cells that were color coded off-blue (light gray) in (a,b) are colored blue (dark gray).

The oxygen speed is defined in the model as the number of layers that the front advances per time unit, defining a layer as the number of cells divided by the width of the system. Hence, $v x L$ cells will change their state from 0 to 1 at random locations of the oxygen front. The lateral diffusion of oxygen along the front is modelled in the following way: Any column must have a total difference in height of less than 2 cells with adjacent columns. When this difference is larger (e.g. third column Fig. 5b), this particle diffuses along the surface, to the next column (second column in Fig. 5c).

The particle diffuses to the side where the maximum different of heights is observed (Fig. 6a). It can occur that the difference with both of the sides is the same. Then the particle diffuses randomly to its left or right column (Fig. 6b). Figures 5 and 6 demonstrates that the effect of the lateral diffusion is for smoothen the front.

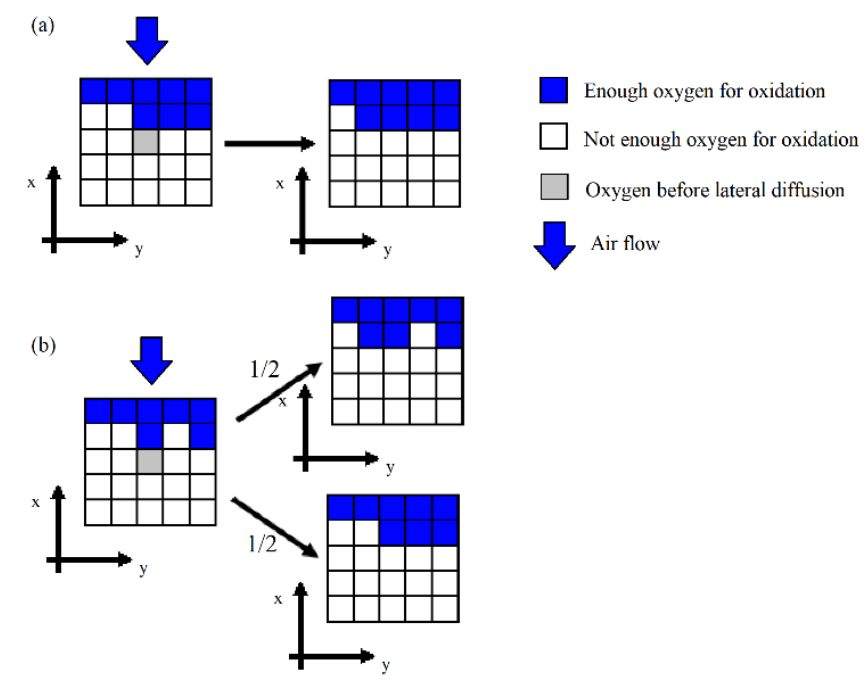

FIG. 6. Lateral diffusion along the oxygen front. (a) The diffusion occurs to the side with greatest height difference with the site, to the left in this case. (b) If both sides have the same difference, diffusion will occur to each of the sides with probability $1 / 2$. 
The oxygen consumed by an oxidation depends both on the fuel and on the conditions of the process (temperature, pressure, etc.). This dependence is represented by the size of the so-called oxygen shadow (Fig. 7). The oxygen shadow is the area where oxygen has been consumed by oxidation and no further oxidation will occur until the oxygen front has arrived at this point again [19].

(a)

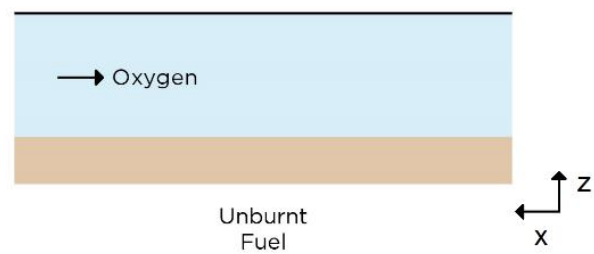

(c)

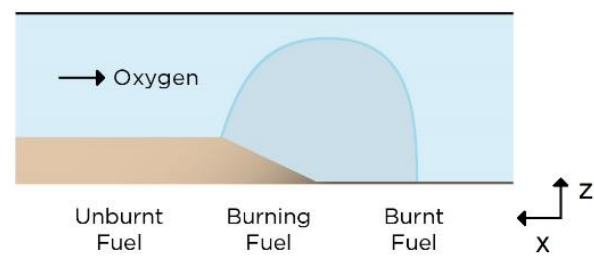

(b)

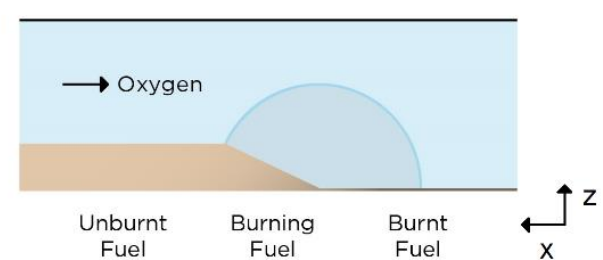

(d)

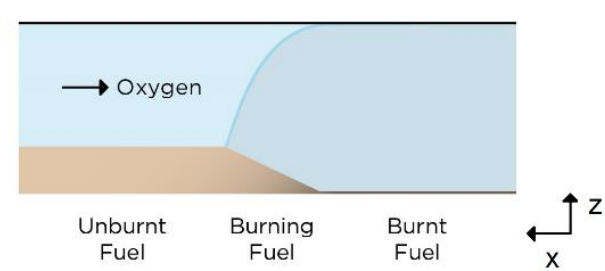

FIG. 7. Schematics of the formation of an oxygen shadow. Opposed smoldering is represented, with the direction of the smoldering front in the positive $\mathrm{x}$ direction and the oxygen flow in the negative $\mathrm{x}$ direction. Gray color represents the inner part of the oxygen shadow, where oxygen is not sufficient for oxidation. (a-d) represent a sequence in time of a growing oxygen shadow. Courtesy of Belen Otero, 2017.

Smoldering is an oxygen-limited process. In our cellular automaton, when oxidation occurs in a cell in the fuel layer the corresponding cell from the oxygen layer and its surroundings, with a radius equal to $\Theta$, are consumed. After this consumption, the oxygen front diffuses laterally and the oxygen flow can continue, as shown in Fig. 8.
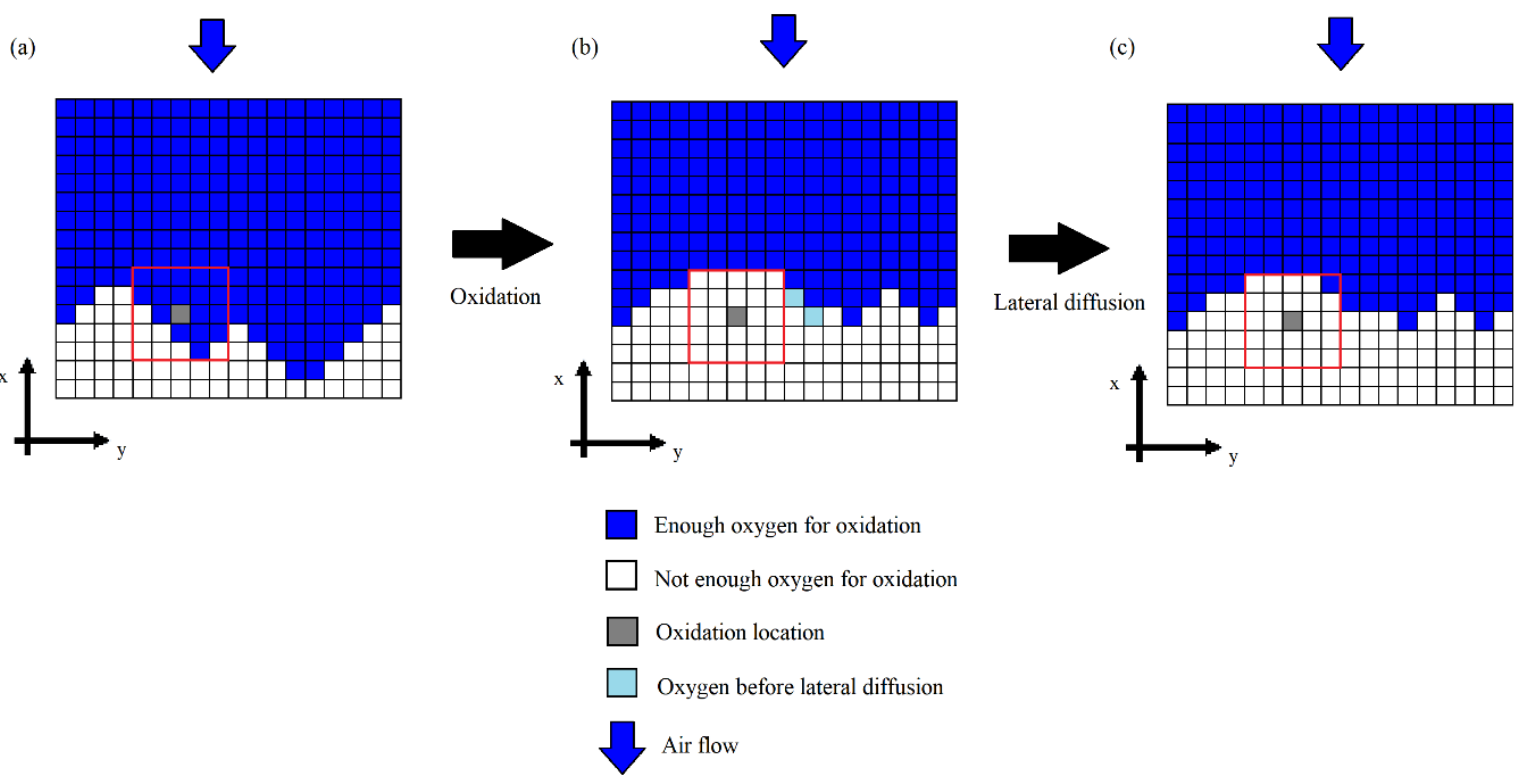

FIG. 8. Sketch illustrating the effect that oxidation has on the oxygen layer. (a,b) Oxidation takes place at the gray site, consuming the oxygen within the red (darker) square of sides $2 \theta$. (b,c) Lateral diffusion will smooth the oxygen front. The radius of the oxygen shadow $\theta$ is two cells. Lateral diffusion occurs after the oxidation. 


\section{Rules of the model}

The three steps that govern the smoldering process are drying, pyrolysis, and oxidation. The changes that these steps cause in the different layers are given by the rules in section II-A. Note that the equations associated with the layers do not take into account the exchanges of heat that take place at a specific space and time during oxidation, pyrolysis and drying. We address these occurrences in the rules of the model specified below.

$\mathbf{1}^{\text {st }}$ step. Drying is removing the water contained in the fuel by applying a certain amount of heat. The content of moisture in the fuel is one of the most important characteristics affecting the probability of occurrence and spread of smoldering [20],

$$
\text { Fuel }(\text { wet })+\text { Heat } \rightarrow \text { Fuel }(\text { dry }) .
$$

The rule governing this step assumes that if a wet fuel cell has enough heat in the heat layer for drying (endothermic heat of drying $-\Omega_{\mathrm{d}}$ ), then this cell is going dry with a probability $\mathrm{p}_{\mathrm{d}}$ in any time step. Changes then occur in the fuel and in the heat layers.

$$
\begin{aligned}
F_{i, j}^{t}=0 & \rightarrow F_{i, j}^{t+1}=1, \\
H_{i, j}^{t} \geq \Omega_{d} & \rightarrow H_{i, j}^{t+1}=H_{i, j}^{t}-\Omega_{d},
\end{aligned}
$$

where $F_{i, j}^{t}$ represents the state of the (i,j) cell of the fuel layer in the time $\mathrm{t}, F_{i, j}^{t+1}$ the state of the (i,j) cell of the fuel layer in the time $\mathrm{t}+1, H_{i, j}^{t}$ the state of the (i,j) cell of the heat layer in the time $\mathrm{t}$ and $H_{i, j}^{t+1}$ the state of the $(i, j)$ cell of the heat layer in the time $t+1$.

$2^{\text {nd }}$ step. Pyrolysis, the thermochemical decomposition of organic material at elevated temperatures without consumption of oxygen and forming char,

$$
\text { Fuel (dry) + Heat } \rightarrow \text { Char. }
$$

The rule governing this step assumes that if a dry fuel cell has enough heat to undergo pyrolysis (endothermic heat of pyrolysis $-\Omega_{\mathrm{p}}$ ), then it will pyrolise with a probability $\mathrm{p}_{\mathrm{p}}$ within a unit time-step. This step involves changes in the fuel and in the heat layers,

$$
\begin{aligned}
F_{i, j}^{t}=1 & \rightarrow F_{i, j}^{t+1}=2, \\
H_{i, j}^{t} \geq \Omega_{p} & \rightarrow H_{i, j}^{t+1}=H_{i, j}^{t}-\Omega_{p} .
\end{aligned}
$$

$3^{\text {rd }}$ step. Oxidation, a thermochemical reaction at elevated temperatures that consumes oxygen and produces heat,

$$
\text { Char }+ \text { Oxygen } \rightarrow \text { Ash }+ \text { Heat }
$$

The rule governing this step assumes that if a char cell has enough oxygen to undergo oxidation - this cell is going to be oxidized with a probability $p_{p}$ within a unit time-step, releasing a specific amount of heat (exothermic heat of oxidation $-\Omega_{\mathrm{o}}$ ). Changes then occur in all three layers.

$$
\begin{aligned}
F_{i, j}^{t}=2 & \rightarrow F_{i, j}^{t+1}=3, \\
H_{i, j}^{t}=H_{i, j}^{t} & \rightarrow H_{i, j}^{t+1}=H_{i, j}^{t}+\Omega_{o}, \\
O_{i, j}^{t}=1 & \rightarrow O_{i, j}^{t+1}=0,
\end{aligned}
$$

where $O_{i, j}^{t}$ represents the state of the $(i, j)$ cell of the oxygen layer in the time t, $O_{i, j}^{t+1}$ the state of the $(i, j)$ cell of the oxygen layer in the time $t+1$. 


\section{E. Parameters of the model}

The inputs of the model consists of 13 parameters that represent the governing parameters of the three layers (Table 1). The oxygen speed and the gap of the Hele-Shaw apparatus, through the Peclet number, are the parameters that control the behavior of the fingering phenomenon. In this article, 11 of these parameters' values have been fixed according to previous studies to a set of values that gave reasonable results and based on the properties of the studied fuel $[14,21]$. Here we focus on the influence of the speed of the oxygen $v$ and the radius of the oxygen shadow $\Theta$ on the fingering phenomenon.

TABLE I. Parameters of the model

\begin{tabular}{ccc}
\hline Parameter & Nomenclature & Value used \\
\hline Length of the sample & $\mathrm{L}$ & 100 \\
Width of the sample & $\mathrm{W}$ & 100 \\
Probability of drying & $\mathrm{p}_{\mathrm{d}}$ & 0.5 \\
Probability of pyrolysis & $\mathrm{p}_{\mathrm{p}}$ & 0.5 \\
Probability of oxidation & $\mathrm{p}_{\mathrm{o}}$ & 0.5 \\
Heat of drying & $\Omega_{\mathrm{d}}$ & 0.05 \\
Heat of pyrolysis & $\Omega_{\mathrm{p}}$ & 0.05 \\
Heat of oxidation & $\Omega_{\mathrm{o}}$ & 1 \\
Heat transfer parallel to the oxygen flow & $\gamma_{\mathrm{f}}$ & $0.05+0.05 v^{1 / 2}$ \\
Heat transfer antiparallel to the oxygen flow & $\gamma_{\mathrm{o}}$ & $0.2 \gamma_{\mathrm{f}}$ \\
Heat transfer perpendicular to the oxygen flow & $\gamma_{1}$ & $\frac{\gamma_{f}+\gamma_{o}}{2}$ \\
Speed of the oxygen flow & $v$ & Variable \\
Radius of the oxygen shadow & $\Theta$ & Variable \\
\hline \hline
\end{tabular}

\section{F. Data processing}

Typical results obtained from the model are as shown on the left of Fig. 9. For image processing purposes, we consider only cells in the ash state as belonging to fingers, so the data was treated as shown in the right of Fig. 9. 


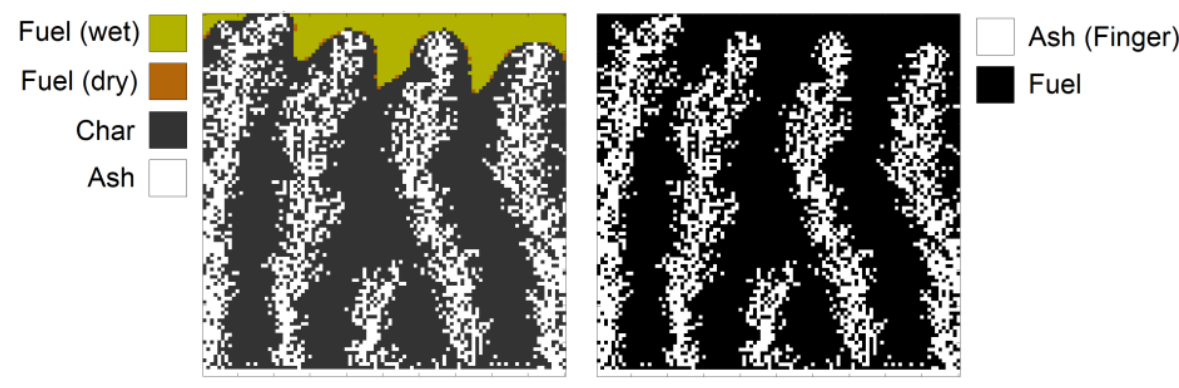

FIG. 9. Four-state structure obtained with the cellular automaton described, left, and corresponding finger structure, right. Only cells in the ash state are considered as part of the fingers, while cells in any of the other three states (wet fuel, dry fuel, and char) are not.

The characteristics determined from these results are the number of fingers, the distance between fingers, the width of the fingers, and the time needed for the first finger to reach the far end of the domain. To determine the first characteristics, a three-step post-processing of the data was necessary, as showed in Fig. $10(a \rightarrow b)$ filling the gaps in the fingers; $(b \rightarrow c)$ eliminating the outliers; and $(c \rightarrow d)$ connecting small islands to the fingers. In the first step, the gaps are flood-filled using 4-connected neighbors (imfill function in Matlab® software). The clusters smaller than 6 cells were eliminated in the second step, ensuring that only groups of more than $5 \%$ of the width/length of the studied area were considered as a part of the proper fingers. Finally, the remaining small islands are connected to the fingers if they are less than 1 cell away, considering that they are part of these fingers.

(a)

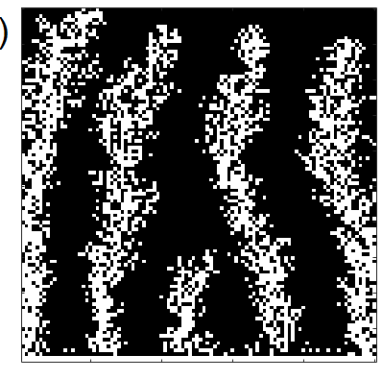

(c)

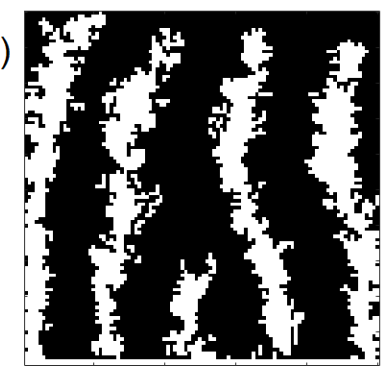

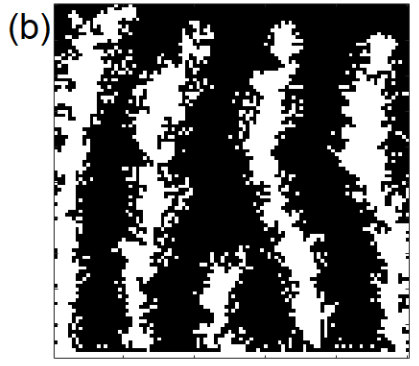

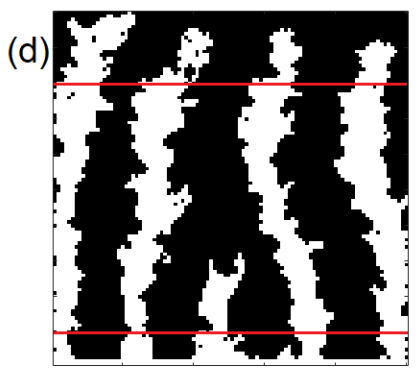

FIG. 10. Figure processing to find fingers and their characteristics. From left to right and from top to bottom: (a) result of the cellular automaton with only two states (Fig. 9, right); (b) enclosed gaps filled; (c) clusters of less than six cells were eliminated; (d) isolated cluster were connected to the main fingers. Structures as obtained in (d) were further analyzed.

The number of fingers is determined as an average of the number of fingers per row, from the $10^{\text {th }}$ to the $80^{\text {th }}$ row to avoid the effect of boundaries, as the number of times the fuel matrix changes from 1 (finger) to 0 (paper). The number of cells at value 0 were counted. This number when divided by the number of fingers minus 1 gave the distance between fingers. The finger width was determined as the number of cells filled (at value 1) divided by the number of fingers. By averaging the values obtained over rows 10 to 80, the number of fingers, the distance between them and the finger width for each speed and radius of the oxygen shadow were obtained. When the number of fingers was less than 2 , the 
smoldering front was considered smooth (no fingers), so the width of the fingers was set to 100 and the distance between fingers to 0 .

\section{INFLUENCE OF THE OXYGEN SPEED}

The oxygen speed influences the occurrence and growth of instabilities of the smoldering front in two different ways. First, via oxidation because oxygen transport is a limiting step in smoldering, and therefore decreasing the oxygen speed, the transport of oxygen decreases, hindering the possibility of simultaneous oxidation processes in different locations. This decrease in the number of cells where oxidation can happen produces a decrease in the number of splits in the fingers, and the distance between fingers increases. Second, the overall heat transfer decreases when the oxygen speed is reduced. Thus, heat transfer is reduced, with decreased levels of drying and pyrolysis. This leads to a decrease in the width of the fingers.

The large variation in patterns generated as the imposed oxygen speed changes is shown in Fig. 11.

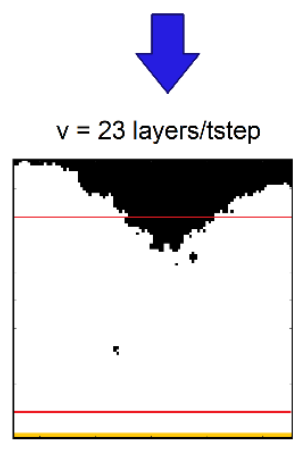

$t=709$

$v=15$ layers/tstep

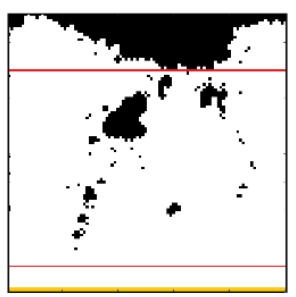

$t=907$

$v=7$ layers/tstep

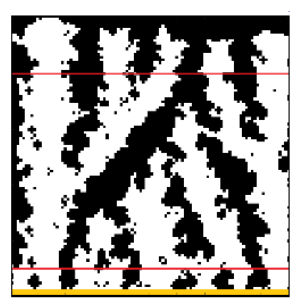

$t=1169$

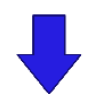

$v=21$ layers/tstep

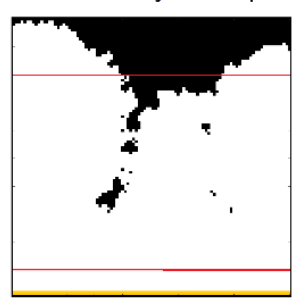

$\mathrm{t}=730$

$v=13$ layers/tstep

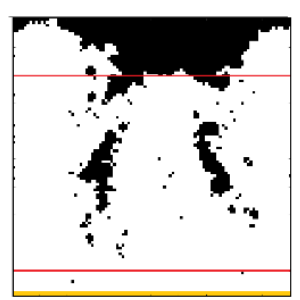

$t=961$

$v=5$ layers/tstep

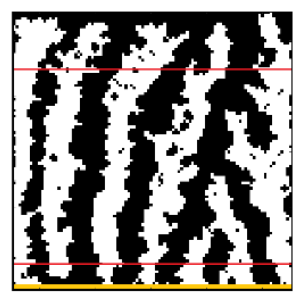

$t=1378$

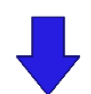

$v=19$ layers/tstep

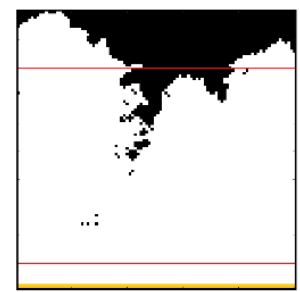

$t=765$

$v=11$ layers $/$ tstep

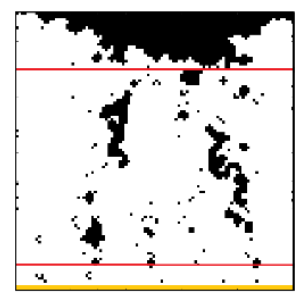

$t=1080$

$\mathrm{v}=3$ layers/tstep

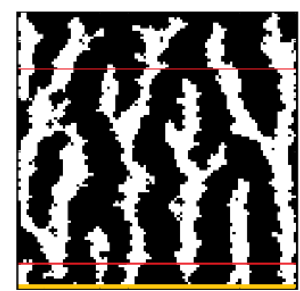

$t=1590$

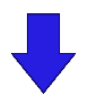

$v=17$ layers/tstep

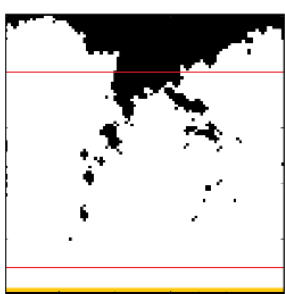

$t=835$

$\mathrm{v}=9$ layers $/$ tstep

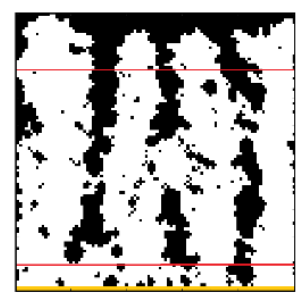

$t=1105$

$v=1$ layer $/$ tstep

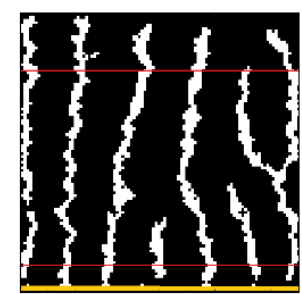

$t=2424$

FIG. 11. Patterns generated as the oxygen speed is varied, for an oxygen shadow radius equal to ten cells, at the time step expressed behind each pattern. As the oxygen speed increases, the width of the fingers increases and the distance between them decreases, until the fingers disappear and a smooth continuous front is observed. The arrows represent the direction of the oxygen flow. Initial ignition takes place along the yellow line at the bottom edge. 
Starting from the top left part of Fig. 11, when the oxygen speed is high, the ignition has plenty of oxygen to ensure oxidation, it causes a smooth and continues front (no fingers are formed).

If the speed decreases beyond a critical value, fingers appear due to the depletion of oxygen near the smoldering front. When oxidation takes place, the oxygen consumed causes an oxygen shadow, and a period of time follows with insufficient oxygen available downstream of the oxidizing cells. As the oxygen increases, it arrives at the locations where all the conditions for oxidation are present. At the beginning of the smoldering process, all the locations where oxidation can take place are in a horizontal line, so the process can start anywhere along this line. However, once fingers start appearing, the oxygen flow reaches the tips of the fingers before any other location, favoring its propagation and preventing the oxidation at other point downstream.

In these cases, the overall heat transfer coefficients are relatively high, due to their direct relation with the oxygen speed, thus, a larger area will be preheated by the oxidation, which induces lateral growth of the fingers.

If the speed decreases even more, bottom-right situations on Fig. 11, sparse fingers are observed. The access to oxygen away from the fingers is even more limited, and only some sparse locations at the finger tips allow oxidation. The overall heat transfer coefficient is low, causing a limited transfer of heat which also limits the drying and the pyrolysis process, leading to thin fingers.

\section{INFLUENCE OF THE OXYGEN CONSUMPTION}

The size of the oxygen shadow strongly influences the behavior of the instabilities observed at the fire front. Depending on this parameter, the number of fingers, the type of fingers and the time they appear differs.

For the smallest radius studied $(\Theta=5)$, the case of the sparse fingers is not observed. Even for the lowest oxygen speed, the fingers display tip-splitting, see Fig. 12.

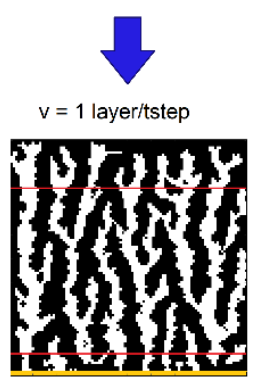

$t=1592$

$v=0.5$ layers/tstep

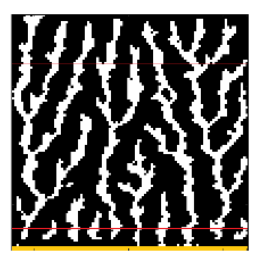

$\mathrm{t}=2253$

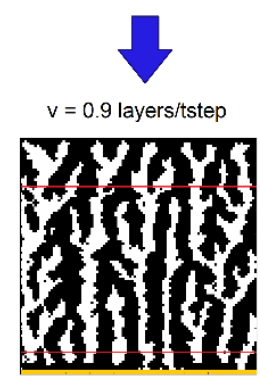

$t=1692$

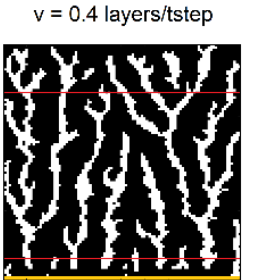

$t=2508$

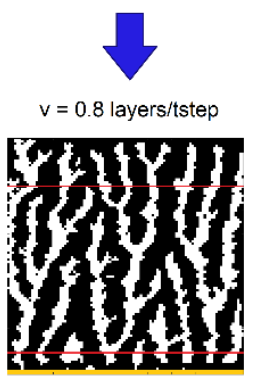

$t=1789$

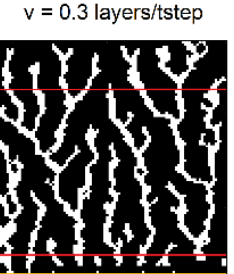

$t=3094$

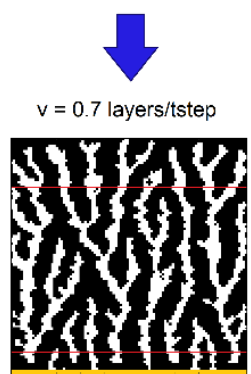

$t=1901$

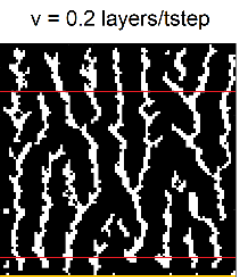

$t=4235$

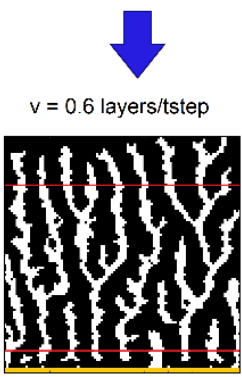

$t=2013$

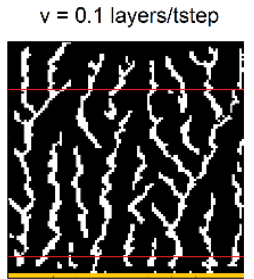

$t=7368$

FIG. 12. Fingers patterns for an oxygen shadow radius of five cells, at the time step expressed behind each pattern. The lowest oxygen speed already produces a tip-splitting pattern, and no appearance of isolated fingers is observed in this case. The arrows represent the direction of the oxygen flow. Initial ignition takes place along the yellow line at the bottom edge. 
Comparing the different radii studied, the number of fingers decreases when the radius increases. The transition from simple fingers to tip-splitting fingers, and to a smooth continuous front occurs at lower oxygen speeds when the radii are smaller, see Fig. 13.

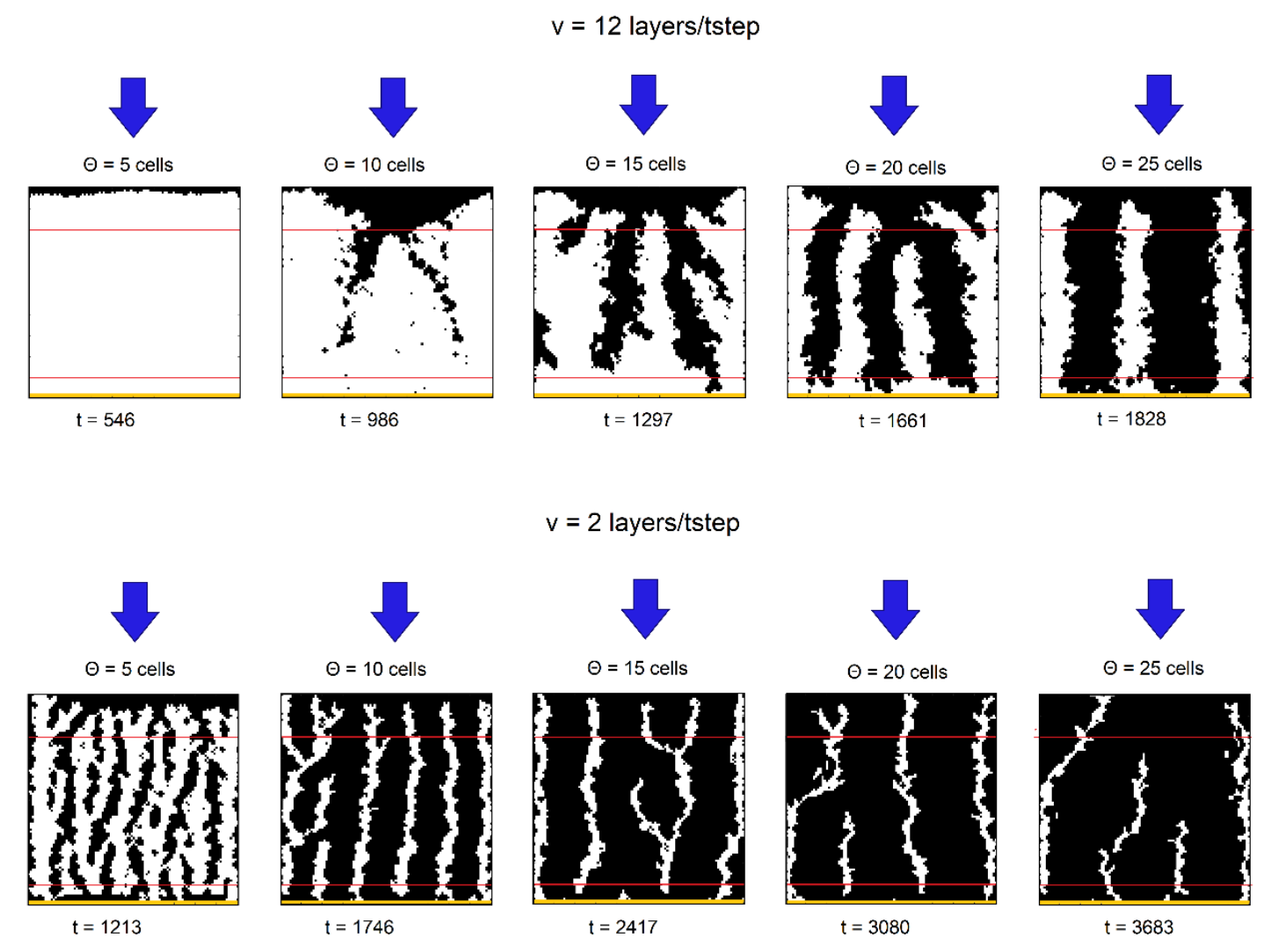

FIG. 13. Evolution of the patterns for two oxygen speeds; the top five correspond to two layers per time step and the bottom five correspond to 12 layers per time step, at the time step expressed behind each pattern. At low oxygen speed, for the five studied oxygen shadow sizes fingers appear. At higher speed, the smallest radius produces a continuous front, while the highest radius produces isolated fingers. The arrows represent the

direction of the oxygen flow. Initial ignition takes place along the yellow line at the bottom edge.

The amount of oxygen consumed during oxidation influences the oxygen speed needed for the occurrence of a smooth smoldering front. For the smallest size studied, transitions take place at lower speeds and the smooth front is observed for relatively large oxygen velocities, more than two times the oxygen shadow radii. On the other hand, the largest size studied present well-defined fingers until relatively large speeds are reached.

\section{QUANTITATIVE ANALYSIS}

Zik et al. [8] established that the instabilities observed in the smoldering front are controlled by the Peclet number. The Peclet number is defined as $P e=\frac{v \cdot h}{D}$, where $v$ is the oxygen speed, $h$ is the height of the gap of the Hele-Shaw apparatus and $D$ is the oxygen diffusivity. $D$ can be considered constant (neglecting the temperature dependence) and we control the oxygen speed through the input parameter $v$. The space between the plate and the glass in the Hele-Shaw apparatus cannot be controlled in the model, due to its two-dimensional nature. However, considering that the amount of oxygen consumed (volume of the oxygen shadow) during any oxidation is proportional to the number of cells included in 
the square centered in the oxidation location with $2 \Theta$ per side, the volume of the oxygen consumed is proportional to $h$ and $\Theta$ squared. With this relation, we can conclude that, for our model, $P e \propto \frac{v}{\Theta^{2}}$.

In order to study the repeatability of the fingering behavior with cellular automata, we have multiplied the number of fingers by the distance between fingers for each row (from $10^{\text {th }}$ to $80^{\text {th }}$ ) and plot them in a staircase diagram (blue line in Figure 14) with the average value represented as the red horizontal line. We have also calculated the difference between the particular value in each row and the average, and plot the results in histograms that show the existing variability on the obtained results, as well as the good agreement existing between the different repetitions. As an example, results obtained for $\Theta$ equal to 10 cells and an oxygen speed of 5 layers per time step are shown in Figure 14.
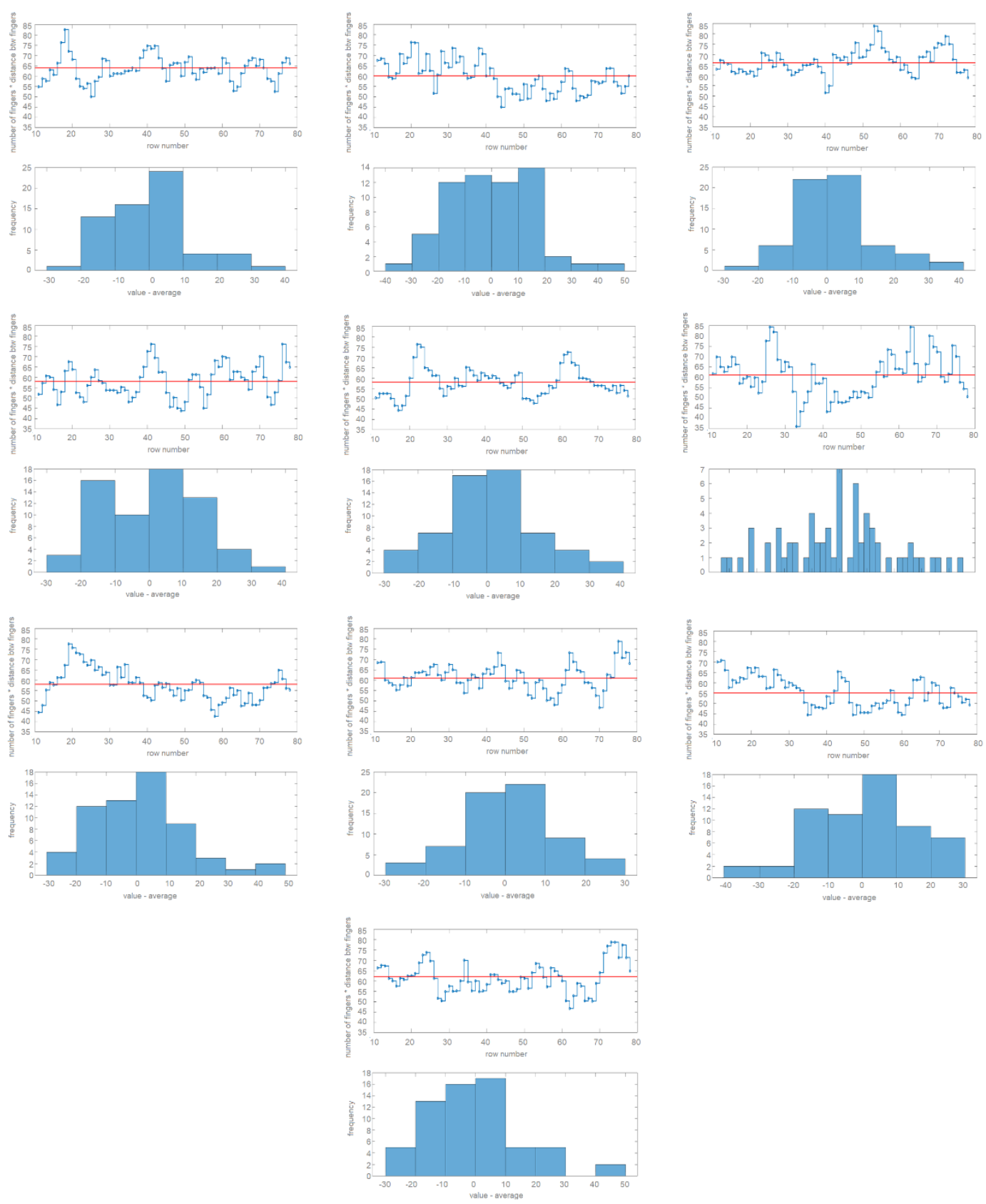

FIG. 14. Staircase diagrams and histograms of the values of the distance between fingers times the number of fingers for $\theta$ equal to ten cells and oxygen speed equal to five layers per time step. Each one of the pairs of graphs (staircase and histogram) is a repetition of the simulation with these values. The red (gray) line in the staircase diagrams represents the average value for the rows from tenth to 80th. 
Experimental results showed that by increasing Pe, the distance between fingers decreases, abruptly at the beginning and slower later on, until a point where it becomes constant for high values of Pe, where a smooth front is present. We observed a decrease in the distance between fingers with $v / \Theta^{2}$. However, the maximum distance between fingers, as well as the number of fingers, heavily depends on the radius of the oxygen shadow. By multiplying these parameters, the distance between fingers and the number of fingers, one obtains a quantity that falls off with oxygen speed in a similar way for all the oxygenshade values, as shown in Fig. 15 (a). These results show the same trend than the experimental results from [8]: a first, abrupt, decrease of the number of fingers $\mathrm{x}$ distance between them, followed by a slower decrease, and finishing with a constant horizontal slope for high values of $v / \Theta^{2}$. The change of slope from an abrupt decrease to a slower one takes place when the air speed equals the oxygen shadow radius $(\Theta)$, and the smooth front behavior starts when the air speed equals two times the oxygen shadow radius $(2 \Theta)$. In the case of $\Theta$ equal to 5, the distinction between a smooth front and extremely closed wide fingers is not possible, so the model considers that for this radius, there is smooth front since the air speed is equal to $\Theta$.

(a)
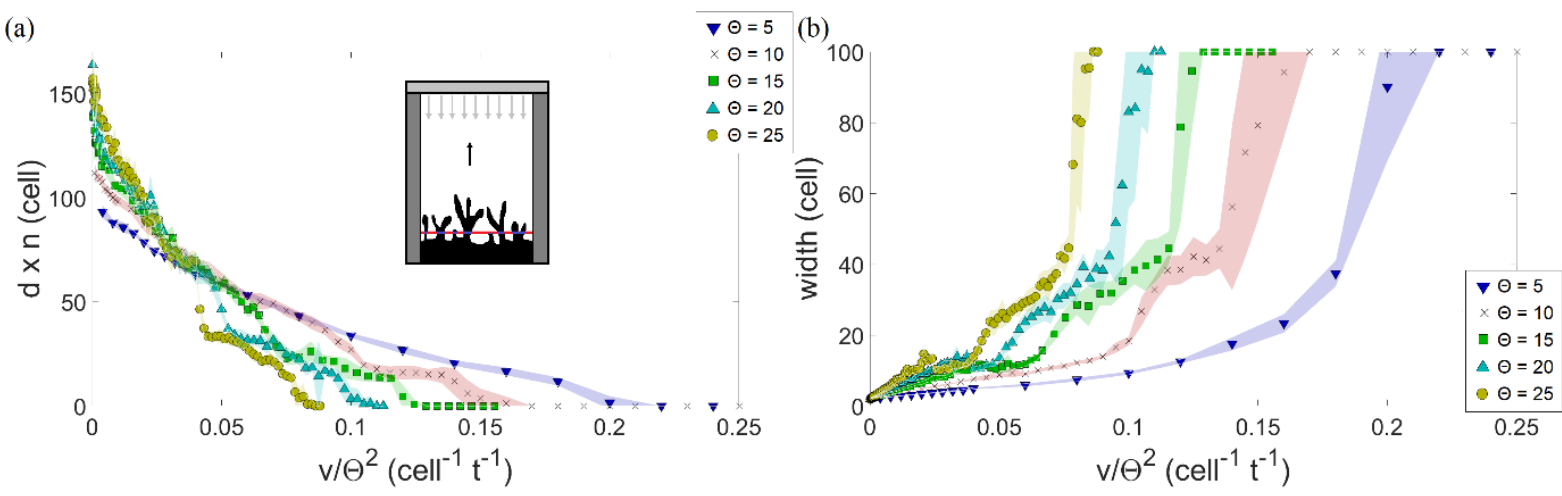

FIG. 15. (a) Distance between fingers times the number of fingers versus oxygen speed for different oxygen shadow sizes; (b) width of the fingers versus oxygen speed for different oxygen shadow sizes. The dots show the average (ten simulations) of the different oxygen speed values used in the simulation, while the shadow represents the standard deviation of the results. The inset schematic shows the modeled process, with the directions of the air flow and the smoldering spread, and a horizontal line showing fingers (blue) and distance between fingers (red).

Figure 15 shows (a) the number of fingers $x$ distance between fingers and (b) the width of the fingers vs $v / \Theta^{2}$. The dots represent the average value of the 10 simulations done for each pair of $v$ and $\Theta$ values, while the shadow is the standard deviation of these values, obtained with Eq. (14).

$$
\sigma_{n-1}^{2}=s^{2}=\frac{1}{N-1}\left[\sum_{i=1}^{N} x_{i}^{2}-\frac{1}{N}\left(\sum_{i=1}^{N} x_{i}\right)^{2}\right]
$$

The width of the fingers, Fig. 15 (b), contrary to the preliminary results from the experimental work of [8], increases with increasing Peclet number. This can be explained by the influence of the oxygen speed on this width. When the oxygen speed increases, the overall heat transfer parameter increases $\left(\gamma_{m}=B+A v^{1 / 2}\right)$. This increase causes a further spread of heat to the neighborhood, favoring the smoldering process to reach further locations and generating wider fingers. This shows the dependency of the width of the fingers with the heat transfer processes that take place during the smoldering combustion. The dependency on the oxygen shadow radii can be observed in this case too: the width of the fingers increases in a not very pronounced slope until the air speed equals the radius of the oxygen shadow $(\Theta)$. In this point, it increases quicker until the air speed equals two times the oxygen shadow $(2 \Theta)$, where only smooth fronts are present, and the width of the fingers is consider as the width of the fuel sample (100 cells).

\section{CONCLUSIONS}


A multi-layer cellular automaton has been developed to model smoldering fires in two dimensions and has been used to mimic a specific behavior that may occur in thin materials: fingering instabilities at the fire front. The parameters that define generated patterns are the distance between fingers and their width, and the oxygen availability controls them through both the oxygen speed and the oxygen consumed during the oxidation process. Both characteristics, distance between fingers and width of the fingers, are related and both of them vary when the oxygen speed changes. This model demonstrates that lower oxygen availability produces more isolated and thinner fingers, while a large oxygen availability ensures a smooth smoldering front.

This cellular automaton reproduces the complex behavior of fingering instabilities through simple rules with low computational cost, confirming the influence of the oxygen speed and the heat losses previously observed experimentally. The non-dimensionality of the model makes it generic and suitable for being used to investigate the smoldering process in any thin material.

\section{ACKNOWLEGMENTS}

The authors would like to thank the Research Council of Norway, project 238329: Emerging Risks from Smoldering Fires (EMRIS - collaboration) for funding and collaborators from EMRIS project for their support. Special thanks to Nils Roenner and Matthew Bonner from Hazelab at Imperial College London for their valuable comments on the work.

\section{REFERENCES}

[1] P. G. Saffman and F. R. S. S. G. Taylor, "The penetration of a fluid into a porous medium or Hele-Shaw cell containing a more viscous liquid," Proc. R. Soc., vol. 245, no. 1242, pp. 312329, 1958.

[2] L. Paterson, "Radial fingering in a Hele Shaw cell," J. Fluid Mech., vol. 113, pp. 513-529, 1981.

[3] G. Tryggvason and H. Aref, "Numerical experiments on Hele Shaw flow with a sharp interface," J. Fluid Mech., vol. 136, pp. 1-30, 1983.

[4] H. Zhao and J. V. Maher, "Viscous-fingering experiments with periodic boundary conditions," Phys. Rev. A, vol. 42, no. 10, pp. 5894-5897, 1990.

[5] J. I. Wakita, I. Ràfols, H. Itoh, T. Matsuyama, and M. Matsushita, "Experimental Investigation on the Formation of Dense-Branching-Morphology-Like Colonies in Bacteria," Journal of the Physical Society of Japan, vol. 67, no. 10. pp. 3630-3636, 1998.

[6] T. J. Ohlemiller, Smoldering combustion. Center for Fire Research, 1986.

[7] G. Rein, "Smoldering combustion," in SFPE Handbook of Fire Protection Engineering, 2016, pp. 581-603.

[8] O. Zik, Z. Olami, and E. Moses, "Fingering Instability in Combustion," Phys. Rev. Lett., vol. 81, no. 1, pp. 3868-3871, 1998.

[9] K. Kuwana, K. Suzuki, Y. Tada, and G. Kushida, "Effective Lewis number of smoldering spread over a thin solid in a narrow channel," Proc. Combust. Inst., vol. 36, no. 2, pp. 32033210, 2017.

[10] D. Version, "Pattern Formation in Reverse Smoldering Combustion: a Homogenization Approach," no. May, 2012. 
[11] E. R. Ijioma, A. Muntean, and T. Ogawa, "Effect of material anisotropy on the fingering instability in reverse smoldering combustion," Int. J. Heat Mass Transf., vol. 81, pp. 924-938, 2015.

[12] S. Wolfram, "Cellular automata as models of complexity," Nature, vol. 311, no. 5985, pp. 419-424, 1984.

[13] L. H. Encinas, S. H. White, A. M. del Rey, and G. R. Sanchez, "Modelling forest fire spread using hexagonal cellular automata," Appl. Math. Model., vol. 31, no. 6, pp. 1213-1227, 2007.

[14] N. Fernandez-Anez, K. Christensen, and G. Rein, "Two-dimensional model of smoldering combustion using multi-layer cellular automaton: the role of ignition location and direction of air flow," Fire Saf. J., 2017.

[15] C. M. Belcher, J. M. Yearsley, R. M. Hadden, J. C. McElwain, and G. Rein, "Baseline intrinsic flammability of Earth's ecosystems estimated from paleoatmospheric oxygen over the past 350 million years," Proc. Natl. Acad. Sci., vol. 107, no. 52, pp. 22448-22453, 2010.

[16] I. Karafyllidis and A. Thanailakis, "A model for predicting forest fire spreading using cellular automata,” Ecol. Modell., vol. 99, no. 1, pp. 87-97, 1997.

[17] R. Siegel and J. R. Howell, Thermal radiation heat transfer, Third edit. New York, USA, 1992.

[18] F. K. Incropera, D. P. Dewitt, T. L. Bergman, and A. S. Lavine, Foundations of heat transfer, Sixth edit. John Wiley \& Sons, 2013.

[19] T. Kashiwagi, K. B. McGrattan, S. L. Olson, O. Fujita, M. Kikuchi, and K. Ito, "Effects of slow wind on localied radiative ignition and transition to flame spread in microgravity," Symp. Combust., vol. 26, no. 1, pp. 1345-1352, 1996.

[20] X. Huang, G. Rein, and H. Chen, "Computational smoldering combustion: predicting the roles of moisture and inert contents in peat wildfires," Proc. Combust. Inst., vol. 35, no. 3, pp. 2673-2681, 2015.

[21] N. Fernandez-Anez, K. Christensen, and G. Rein, "Effect of air flow on the spread of forward and opposed smoldering combustion in biomass," in Fire Safety 2017. 